OPEN ACCESS

Edited by:

Sabrina Cipolletta

University of Padova, Italy

Reviewed by:

Rute F. Meneses,

Fernando Pessoa University, Portugal Henri Guise Laurie Rauch,

University of Cape Town, South Africa

*Correspondence: Hideaki Hasuo

hasuohid@hirakata.kmu.ac.jp

Specialty section

This article was submitted to Family Medicine and Primary Care,

a section of the journal

Frontiers in Medicine

Received: 23 May 2019 Accepted: 11 February 2020

Published: 25 February 2020

Citation:

Hasuo H, Kanbara K and Fukunaga $M$ (2020) Effect of Heart Rate Variability Biofeedback Sessions With Resonant

Frequency Breathing on Sleep: A Pilot

Study Among Family Caregivers of

Patients With Cancer.

Front. Med. 7:61

doi: 10.3389/fmed.2020.00061

\section{Effect of Heart Rate Variability Biofeedback Sessions With Resonant Frequency Breathing on Sleep: A Pilot Study Among Family Caregivers of Patients With Cancer}

\author{
Hideaki Hasuo*, Kenji Kanbara and Mikihiko Fukunaga \\ Department of Psychosomatic Medicine, Kansai Medical University, Osaka, Japan
}

Heart rate variability biofeedback (HRV-BF) is used as a skill in psychosomatic medicine, but is not yet established in the field of sleep. The present study aimed to evaluate the effect of HRV-BF with resonant frequency breathing (RFB) on sleep performed once every 2 weeks and the usefulness of practice of RFB using a portable device at home before bedtime. Participants were 69 family caregivers of patients with cancer that felt burdened by nursing care. We conducted a randomized controlled trial with an HRV-BF+Home practice group and an HRV-BF group. HRV-BF with RFB was administered to both groups at our medical institution for up to $30 \mathrm{~min}$ on the experiment days. Home practice involved RFB using a portable device, which was performed at home each day within 20 min before bedtime. Evaluation items were: change ratio of total score of the Pittsburgh Sleep Quality Index (PSQI) at 28 days after the trial started. In total, $52.2 \%$ of participants had insomnia. The two HRV-BF groups had decreased PSQI total scores, which indicated an improvement in PSQI total score near 5.5 on Day 28. The two HRV-BF groups had significantly increased HRV scores on Day 28, and there was correlation between the variation of PSQI total score and the variation of HRV score. The quality of sleep assessed by PSQI scores in the HRV-BF+Home practice group was significantly improved compared with the HRV-BF group on Day $28(p=0.001)$. This suggests HRV-BF may be a useful skill for enhancing sleep among family caregivers of patients with cancer, as well as supporting their autonomic nervous function. Additional actual regular practice of RFB (using a portable device at home before bedtime) may further enhance the effect.

Keywords: sleep, insomnia disorder, family caregiver, self-control, heart rate variability biofeedback, resonant frequency breathing

\section{INTRODUCTION}

Insomnia is a common phenomenon for those who provide informal care for someone living with a life-limiting condition. Insomnia is a typical psychiatric disorder, with a prevalence of $72 \%$ [as measured by the Japanese version of the Pittsburgh Sleep Quality Index (PSQI)] among family caregivers of patients with advanced cancer in Japan (1). Treatment for insomnia disorder is mainly 
medication therapy, but there are problems associated with longterm treatment such as decreased self-efficacy, dependency, and developing tolerance (2). In early palliative care, patients with cancer and family caregivers showed increased use of approachoriented skills, which was associated with higher self-efficacy and quality of life (3).

Biofeedback $(\mathrm{BF})$ is a behavioral therapy that regulates the mind and body by measuring physiological information of which people are normally unaware and visually feeding back this information, which creates greater self-awareness and fosters better skills (4). A protocol for heart rate variability (HRV) BF (HRV-BF) sessions using resonant frequency breathing (RFB) has been established (5) and found useful for people with hyperactive autonomic nervous function, mood disorders, and fibromyalgia (6-8). HRV measures the fluctuation in the interval between heartbeats and reflects autonomic nervous activity. RFB is a method of breathing that maximizes HRV by creating a resonance between breathing and the baroreceptor reflex (5). RFB was found to enhance baroreflex sensitivity among patients with chronic heart failure (9).

Physical functions rest during sleep and breathing and heart rates decrease. HRV-BF with RFB uses respiratory sinus arrhythmia to increase HRV (5). In respiratory sinus arrhythmia, the heart rate increases when breathing in, and decreases when breathing out. It is hypothesized that respiratory sinus arrhythmia is an intrinsic resting function of the cardiopulmonary system (10). Respiratory sinus arrhythmia for healthy persons is reported to increase during non-rapid eye movement sleep (11). Therefore, HRV-BF with RFB may influence resting function during sleep. A previous report found healthy persons could increase respiratory sinus arrhythmia during sleep by HRV-BF before bedtime (12). Moreover, a randomized controlled trial involving an HRV-BF group and a control group investigated the use of a portable device in terms of the first night effect for healthy persons (13). Polysomnography showed that although there was no improvement in sleep latency in the HRV-BF group the following day, the quality of sleep was enhanced. A previous case report suggested that HRV$\mathrm{BF}$ with use of a portable device for 1 week after application could improve insomnia disorder (14). However, few research reports have investigated if $\mathrm{HRV}-\mathrm{BF}$ before bedtime or the practice of RFB could have a medium- to short-term effect on sleep. A controlled before-and-after trial involving patients with posttraumatic stress disorder indicated that HRV-BF with a portable device before bedtime significantly reduced the Insomnia Severity Index 4 weeks after application (15).

To our knowledge, the effect of an HRV-BF session on sleep with RFB has not been reported. A previous study was conducted in which HRV-BF sessions with RFB were performed every 2 weeks for family caregivers of patients with cancer

Abbreviations: HRV-BF, heart rate variability biofeedback; RFB, resonant frequency breathing; PSQI, Pittsburgh Sleep Quality Index; QOL, quality of life; HRV, heart rate variability; BF, biofeedback; J-ZBI, Japanese version of the Zarit Caregiver Burden Interview; LF, low frequency; HF, high frequency; SDNN, standard deviation of the normal-to-normal interval; ANOVA, analysis of variance.
(16). That study showed that after two sessions of HRV-BF, the HRV score increased on Day 28. The effect of the stress burden of lowered autonomic nervous function may reduce respiratory sinus arrhythmia during sleep (17). Therefore, even a short session of HRV-BF may show a positive effect on sleep irrespective of HRV-BF before bedtime or regular practice of RFB. The present study aimed to evaluate the influence of HRV$\mathrm{BF}$ on sleep among family caregivers with a care burden. We aimed to prove the hypothesis that HRV-BF sessions with RFB would have an effect on sleep, and daily practice of RFB before bedtime at home could enhance this effect.

\section{MATERIALS AND METHODS}

\section{Study Design}

We administered HRV-BF with RFB to family caregivers of patients with advanced cancer. We conducted an open-label randomized controlled study involving 69 family caregivers of patients with advanced cancer. The family caregivers were allocated to an HRV-BF+Home practice group or an HRV-BF group with a computer-generated algorithm using minimization methods and a 1:1 allocation ratio, according to whether they performed the RFB method using a portable device at their home (home practice). The HRV-BF+Home practice group performed HRV-BF with RFB at our medical institution on Days 0,14 , and 28 from the start of the intervention. This group also performed RFB using a portable device at home each day before bedtime (home practice). The HRV-BF group performed HRV-BF with RFB at our medical institution on Days 0,14 , and 28 , but did not perform the home practice.

\section{Ethics Statement}

This study was approved by the ethics committee of Kansai Medical University, Japan (No. 2015660). Written informed consent was obtained from all study participants. The procedures performed in this study were in accordance with the 1964 Helsinki Declaration and approved by the Human Research Ethics Committee of our institution. This study was registered with the University hospital Medical Information Network Clinical Trials Registry (approval number: UMIN000021639) on March 27, 2016.

\section{Participants}

This study was conducted from 2015 to 2018 at the Kansai Medical University Hospital. We evaluated the level of nursingcare burden experienced by family caregivers using the Japanese version of the Zarit Caregiver Burden Interview (J-ZBI). Participants with a J-ZBI score $\geq 24$ (which is the cutoff value for depression risk) were eligible for this study (18). During this period, 244 family caregivers of patients with advanced cancer who visited the palliative care department were continuously enrolled in this study. Of these family caregivers, 92 met the study eligibility criteria according to their J-ZBI score. Exclusion criteria were: (1) having a disease that affected the evaluation of autonomic nerve function (e.g., diabetes); and (2) having any comorbidity relating to psychiatric disorders or conditions 


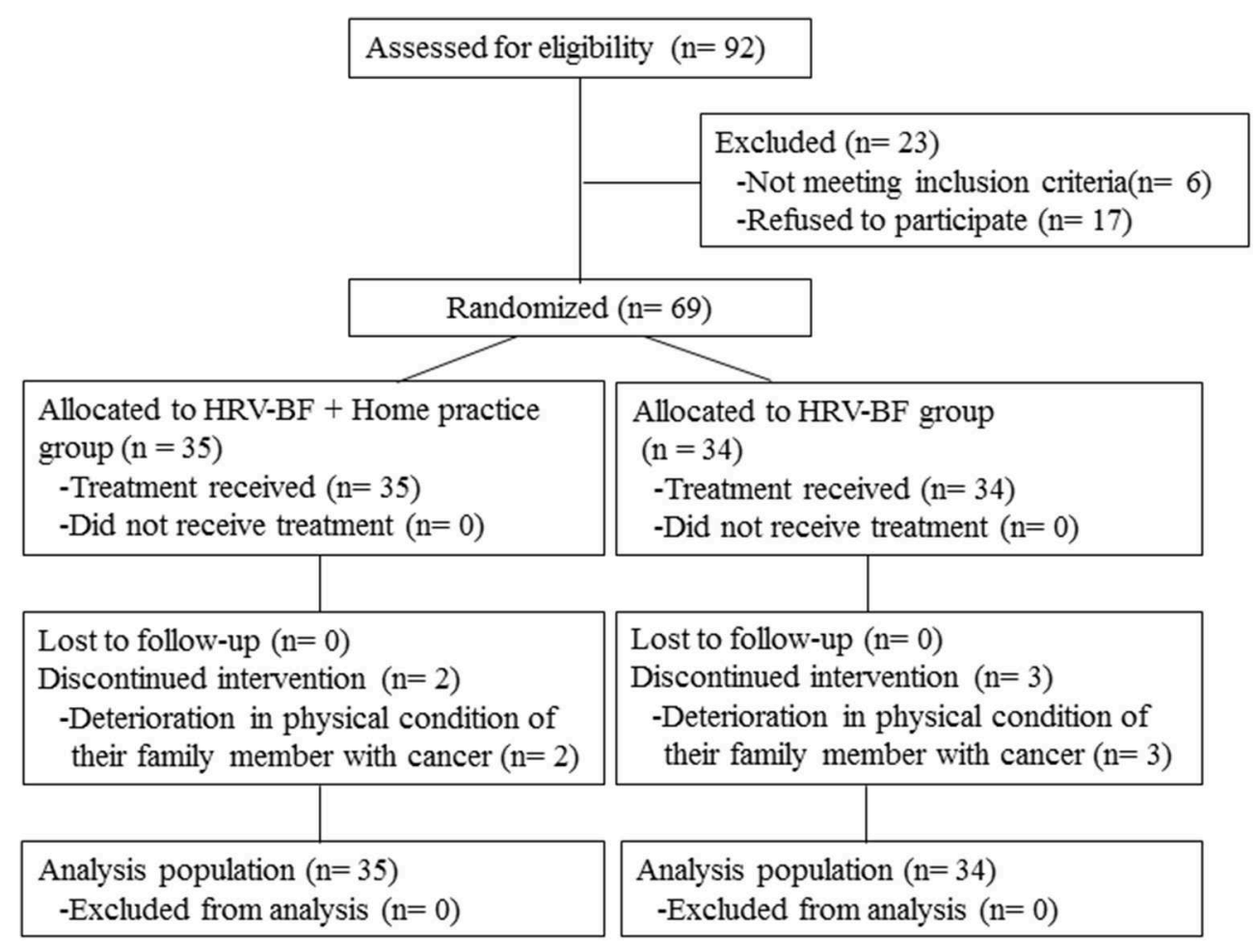

FIGURE 1 | Study flow diagram. HRV, heart rate variability; BF, biofeedback.

that made communication difficult (e.g., cognitive impairment). Figure 1 presents a flow diagram of this study.

\section{Measures}

HRV-BF with RFB was administered to family caregivers of patients with advanced cancer at our medical institution for up to $30 \mathrm{~min}$ on Days 0, 14, and 28 from the start of the intervention. At Day 0, we monitored breathing among participants at our hospital using a multichannel biofeedback system (ProComp Infiniti ${ }^{\mathrm{TM}} /$ BioGraph Infiniti; Thought Technology Ltd., Montreal, Canada) to determine participants' resonant frequency. Participants breathed for $2 \mathrm{~min}$ at $5,5.5,6,6.5$, and 7 breaths per minute while we measured resonant frequency. We calculated the RFB as the number of breaths that maximized the HRV spectrum peak and smoothed the HRV waveform. During HRV-BF, family caregivers used the Breath Pacer application (ProComp Infiniti ${ }^{\mathrm{TM}} / \mathrm{BioGraph}$ Infiniti; Thought Technology Ltd., Montreal, Canada) to maximize the HRV waveform displayed on the screen of a personal tablet computer (iPad mini; Apple, Cupertino, CA, USA). We connected the HRV components (myBeat WHS-2; Union Tool Co., Tokyo, Japan) to an electrode pad attached directly to the participant's chest. The HRV waveform was displayed on the screen in real time.

We downloaded the Breath Pacer application and a breath self-training guide onto portable devices for participants to use at home. Participants were instructed that home practice should be performed each day within $20 \mathrm{~min}$ (or a minimum of
$5 \mathrm{~min}$ ) before going to bed, according to the pace set by their portable device. Breath Pacer data entry was performed based on frequency after the user's RFB had been determined.

We measured HRV before administering HRV-BF. Family caregivers performed RFB for $5 \mathrm{~min}$, resting for $5 \mathrm{~min}$ before and after RFB. We measured the HRV continuously for $15 \mathrm{~min}$. These measurements were recorded on Days 0,14 , and 28. We used HRV analysis software (Kubios HRV version 3.1; Kubios Oy, Kuopio, Finland), which is considered highly reliable for short-term recording $(19,20)$. Furthermore, we evaluated family caregivers' sleep using the Japanese version of the PSQI (PSQI$\mathrm{J}$ ), which is a self-report questionnaire that we administered on Days 0 and 28. The data that support the findings of this study are openly available in figshare.

\section{Study Analytical Parameters}

Analytical parameters included demographic factors, relationship with the patient, resonant frequency, and JZBI scores. We extracted additional information including rate of insomnia disorder, PSQI-J score, and HRV score. The primary outcomes of this study were the change rate in the total PSQI-J score and HRV score at 28 days after the intervention start, based on comparisons between the HRV-BF+Home practice group and the HRV-BF group.

\section{Rate of Insomnia Disorder}

We calculated the rate of insomnia disorder by dividing the family caregivers diagnosed with insomnia disorder by all family 
caregivers. Insomnia disorder was diagnosed when a family caregiver met all of the diagnostic criteria for insomnia in the Diagnostic and Statistical Manual of Mental Disorders, Fifth Edition (21).

\section{PSQI-J}

The PSQI-J is the most frequently used scale to assess insomnia occurring in the past month among family caregivers of patients with advanced cancer (1). The PSQI-J total score comprises seven component scores: sleep quality, sleep latency, sleep duration, habitual sleep efficiency, sleep difficulty, hypnotic use, and daytime dysfunction. Each component score ranges from 0 to 3 . The PSQI-J total score is the total score of all components, with higher scores indicating poorer sleep. A PSQI-J global score cut off point of 5.5 yielded estimations of sensitivity and has been validated in caregivers of oncology patients, with a Cronbach's $\alpha$ of $0.68(22)$.

\section{HRV}

HRV, which is the fluctuation of heartbeat interval, is used as a measure reflecting autonomic nerve activity. A low frequency (LF; 0.04-0.15 Hz) component and high frequency (HF; 0.15$0.4 \mathrm{~Hz}$ ) component are recorded within several minutes of HRV (23). These components are obtained by frequency domain analysis and reflect parasympathetic activity. The standard deviation of the normal-to-normal interval (SDNN) is the standard deviation of the R-R intervals in an electrocardiogram, and is obtained through time domain analysis. A decrease in SDNN indicates a decrease in parasympathetic activity (24). The SDNN reflects all contributions to HRV, including sympathetic activity. The mean value for resting HRV in adults are LF $=519$ $\mathrm{ms}^{2}, \mathrm{HF}=657 \mathrm{~ms}^{2}$, and SDNN $=50 \mathrm{~ms}(25)$.

\section{Statistical Analysis}

We used unpaired $t$-tests for the dependent variables: age, resonant frequency, J-ZBI scores, rate of insomnia disorder, PSQI-J total score (Day 0), and SDNN score (Day 0). We used Pearson's chi-square tests to analyze the dependent variables: sex, PSQI-J $\leq 5.5$ or PSQI-J $>5.5$. Changes in the course of PSQI-J score and SDNN score (Days 0, 14, and 28) were analyzed using one-way repeated measures analysis of variance (ANOVA) for each group. To conduct comparisons between groups, we used time-course as the within-subjects factor and group as a between-subjects factor in two-way repeated measures ANOVA. In the ANOVA, multiple comparisons were corrected using the Bonferroni method. The main analysis was based on the intention-to-treat principle. If participants withdrew from the study, the PSQI-J scores and HRV scores after withdrawal were replaced with scores just before withdrawal. We defined withdrawal from home practice of RFB as missing practice more than twice a week. In addition, Pearson's correlation coefficients between the variation of PSQI-J total score and the variation of HRV score were calculated. A $p<0.05$ was regarded as statistically significant. Statistical analyses were conducted using SPSS version 18.0J for Macintosh (SPSS, Inc. IBM, Chicago, IL).

\section{RESULTS}

Table 1 shows the demographic and clinical characteristics of the study groups. Insomnia disorder was diagnosed in 52.2\% [95\% confidence interval (CI): 40.2-64.2] of participants. No sleepwake disorders other than insomnia disorder were observed. Family caregivers in the HRV-BF+Home practice group had a

TABLE 1 | Comparison of clinical characteristics between the HRV-BF + Home practice, HRV-BF, and control groups.

\begin{tabular}{|c|c|c|c|c|c|}
\hline \multirow[b]{2}{*}{ Age (years), mean (SD) } & \multicolumn{2}{|c|}{$\begin{array}{c}\text { HRV-BF + Home } \\
\text { practice group }(n=35)\end{array}$} & \multicolumn{2}{|c|}{$\begin{array}{l}\text { HRV-BF group } \\
\quad(n=34)\end{array}$} & \multirow{2}{*}{$\begin{array}{c}\boldsymbol{P} \\
0.34\end{array}$} \\
\hline & 64.5 & $(10.5)$ & 61.8 & $(12.6)$ & \\
\hline \multicolumn{6}{|l|}{ Sex, $n(\%)$} \\
\hline Male & 12 & (34) & 11 & (32) & 0.87 \\
\hline Female & 23 & $(66)$ & 23 & (68) & \\
\hline \multicolumn{6}{|l|}{$\begin{array}{l}\text { Relationship with the } \\
\text { patients, } n(\%)\end{array}$} \\
\hline Mother & 1 & $(2.9)$ & 1 & $(2.9)$ & \\
\hline Husband & 11 & $(31.4)$ & 10 & $(29.5)$ & \\
\hline Wife & 19 & $(54.2)$ & 17 & $(50.0)$ & \\
\hline Son & 1 & $(2.9)$ & 1 & $(2.9)$ & \\
\hline Daughter & 3 & (8.6) & 5 & $(14.7)$ & \\
\hline \multicolumn{6}{|l|}{$\begin{array}{l}\text { Conditions of the patients } \\
\text { with advanced cancer }\end{array}$} \\
\hline Outpatient, $n(\%)$ & 32 & (91.4) & 30 & (88.2) & 0.67 \\
\hline $\begin{array}{l}\text { Under cancer } \\
\text { treatment, } n(\%)\end{array}$ & 24 & $(68.6)$ & 25 & $(73.5)$ & 0.94 \\
\hline $\begin{array}{l}\text { Necessity of } \\
\text { nighttime care, } n(\%)\end{array}$ & 5 & $(14.3)$ & 3 & $(8.8)$ & 0.49 \\
\hline \multirow[t]{2}{*}{$\begin{array}{l}\text { Resonant frequency, } \\
\text { mean (SD) }\end{array}$} & 6.0 & $(0.7)$ & 6.3 & $(0.7)$ & 0.18 \\
\hline & $n$ & $\%$ & $n$ & $\%$ & \\
\hline 5 & 5 & $(14.3)$ & 3 & (8.8) & \\
\hline 5.5 & 9 & $(25.7)$ & 6 & $(17.6)$ & \\
\hline 6 & 6 & $(17.1)$ & 6 & $(17.6)$ & \\
\hline 6.5 & 8 & $(22.9)$ & 8 & $(23.6)$ & \\
\hline 7 & 7 & (20.0) & 11 & (32.4) & \\
\hline J-ZBI, mean (SD) & 37.7 & $(15.5)$ & 35.1 & $(14.0)$ & 0.46 \\
\hline Insomnia disorder, n (\%) & 19 & $(54.3)$ & 17 & $(50.0)$ & 0.73 \\
\hline $\begin{array}{l}\text { PSQI-J score (day 0), } \\
\text { mean (SD) }\end{array}$ & 9.8 & (3.0) & 8.6 & (3.9) & 0.15 \\
\hline
\end{tabular}

\begin{tabular}{lccccc}
\hline & $\boldsymbol{n}$ & $\%$ & $\boldsymbol{n}$ & $\%$ & \\
\hline PSQI-J $\leqq 5$ & 4 & $(11.4)$ & 4 & $(11.8)$ & 0.97 \\
PSQI-J > & 31 & $(88.6)$ & 30 & $(88.2)$ & \\
$\begin{array}{l}\text { Heart rate variability, } \\
\text { mean (SD) }\end{array}$ & & & & & \\
LF (day 0) & 250.0 & $(116.7)$ & 284.3 .0 & $(178.9)$ & 0.26 \\
HF (day 0) & 224.2 & $(107.4)$ & 191.2 & $(118.5)$ & 0.36 \\
SDNN (day 0) & 27.4 & $(7.2)$ & 29.6 & $(9.4)$ & 0.72 \\
\hline
\end{tabular}

$H R V-B F$, heart rate variability-biofeedback; J-ZBI, Japanese version of Zarit Caregiver Burden Interview; PSQI-J, Japanese version of the Pittsburgh Sleep Quality Index; LF: low frequency; $S D$, standard deviation. 
bigger burden of nursing care, and tended to have higher PSQI$J$ total scores and lower SDNN scores than the HRV-BF group; however, these differences were not statistically significant. Two family caregivers in the HRV-BF+Home practice group and three in the HRV-BF group withdrew from this study during Days 14-28 (completion rate 94.3 and $91.2 \%$, respectively) because their family member with cancer experienced a decline in physical condition. There were no cases in which home practice of RFB was missed more than twice a week.

A comparison of the PSQI-J total scores between groups showed no significant interaction of time course and group $(p$ $=0.114)$. There was only a main effect in time course $(p<$ 0.001). The total PSQI-J scores of both groups were significantly decreased on Day 28 compared with Day 0. In family caregivers with and without insomnia disorder, there was no interaction between time course and group $(p=0.206$ and $p=0.084$, respectively). There was only a main effect in time course ( $p<$ 0.001 and $p=0.002$, respectively) (Figure 2).

Most of the PSQI-J component scores of both groups were significantly decreased on Day 28 compared with Day 0. The sleep quality score showed a significant interaction of time course and group ( $p=0.001)$. There was also a significant difference in time course between the groups (Day $0, p=0.611$; Day 28, $p<$ $0.001)$. In the other component scores, the interactions of time course and group were not significant. In the HRV-BF+Home practice group, the score for sleep latency was increased, but there was no significant difference between before and after (Table 2).

The HRV scores of both groups, including LF and SDNN, were significantly increased on Day 28 compared with Day 0. There was a significant interaction between time course and group ( $p=0.014)$ in LF between the groups. There was also a significant difference in time course between the groups (day

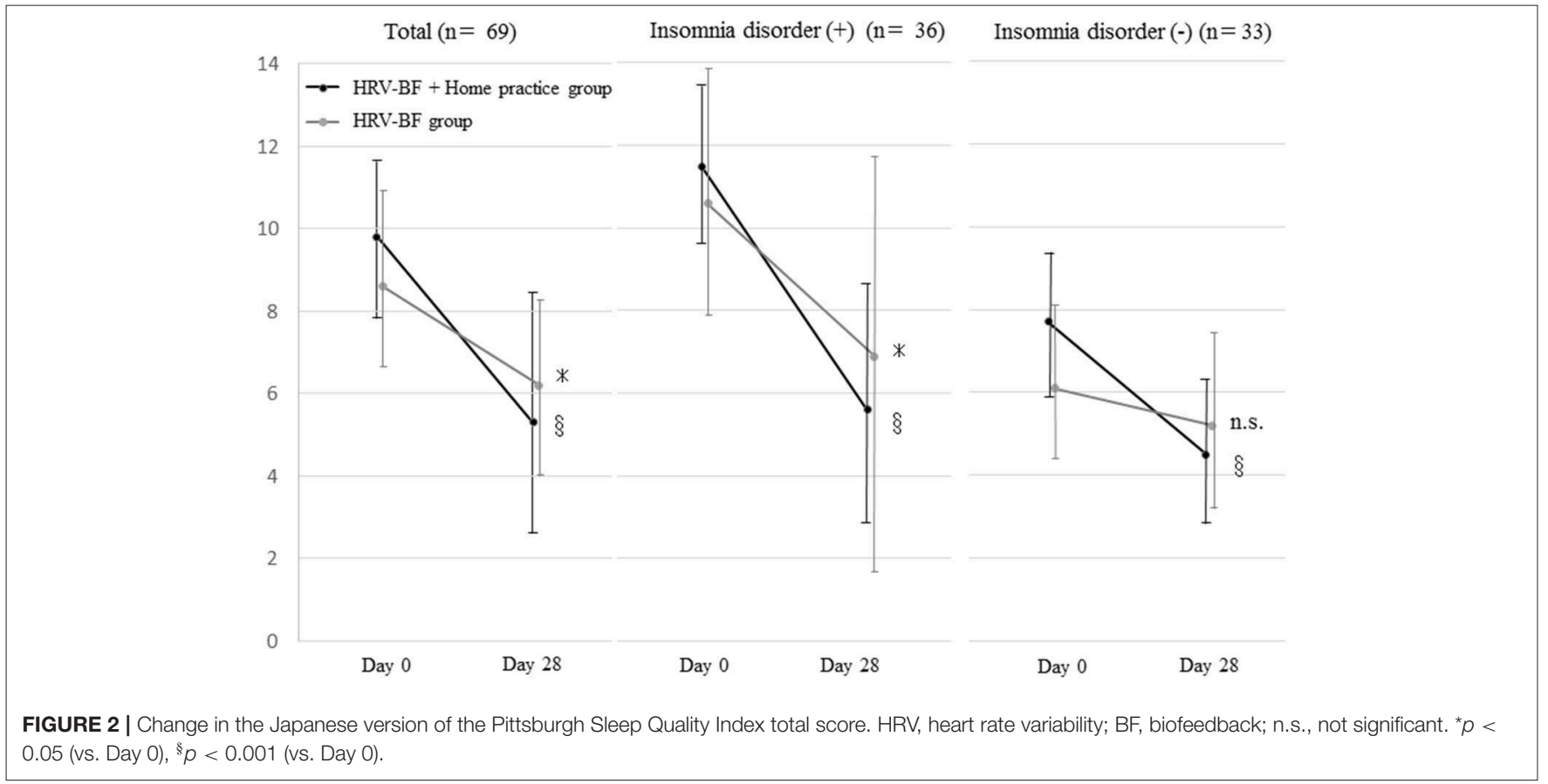

TABLE 2 | Change in the seven component scores of the Japanese version of the Pittsburgh Sleep Quality Index.

\begin{tabular}{|c|c|c|c|c|c|c|c|}
\hline & \multicolumn{3}{|c|}{ HRV-BF + Home practice group } & \multicolumn{3}{|c|}{ HRV-BF group } & \multirow[t]{2}{*}{$P^{b}$} \\
\hline & Mean (SD) & Mean (SD) & $P^{a}$ & Mean (SD) & Mean (SD) & $P^{a}$ & \\
\hline Sleep quality & $19(0.6)$ & $05(0.7)$ & $<0.001$ & $1.8(0.8)$ & $1.2(0.8)$ & 0.003 & 0.001 \\
\hline Sleep latency & $13(0.9)$ & $1.5(1.1)$ & 0.560 & $1.1(1.0)$ & $1.0(1.0)$ & 0.817 & 0.683 \\
\hline Sleep disturbances & $1.0(0.5)$ & $0.5(0.8)$ & 0.001 & $0.8(0.6)$ & $0.8(0.7)$ & 0.852 & 0.062 \\
\hline Use of sleeping medication & $0.5(0.8)$ & $0.3(0.8)$ & 0.305 & $05(0.7)$ & $0.4(1.0)$ & 0.891 & 0.554 \\
\hline Daytime dysfunction & $1.6(1.2)$ & $0.8(0.8)$ & 0.001 & $1.6(1.2)$ & $0.7(0.8)$ & 0.001 & 0.811 \\
\hline
\end{tabular}

a unpaired t-test, ${ }^{b}$ two-way repeated measures analyses of variance. HRV, heart rate variability; BF, biofeedback. 

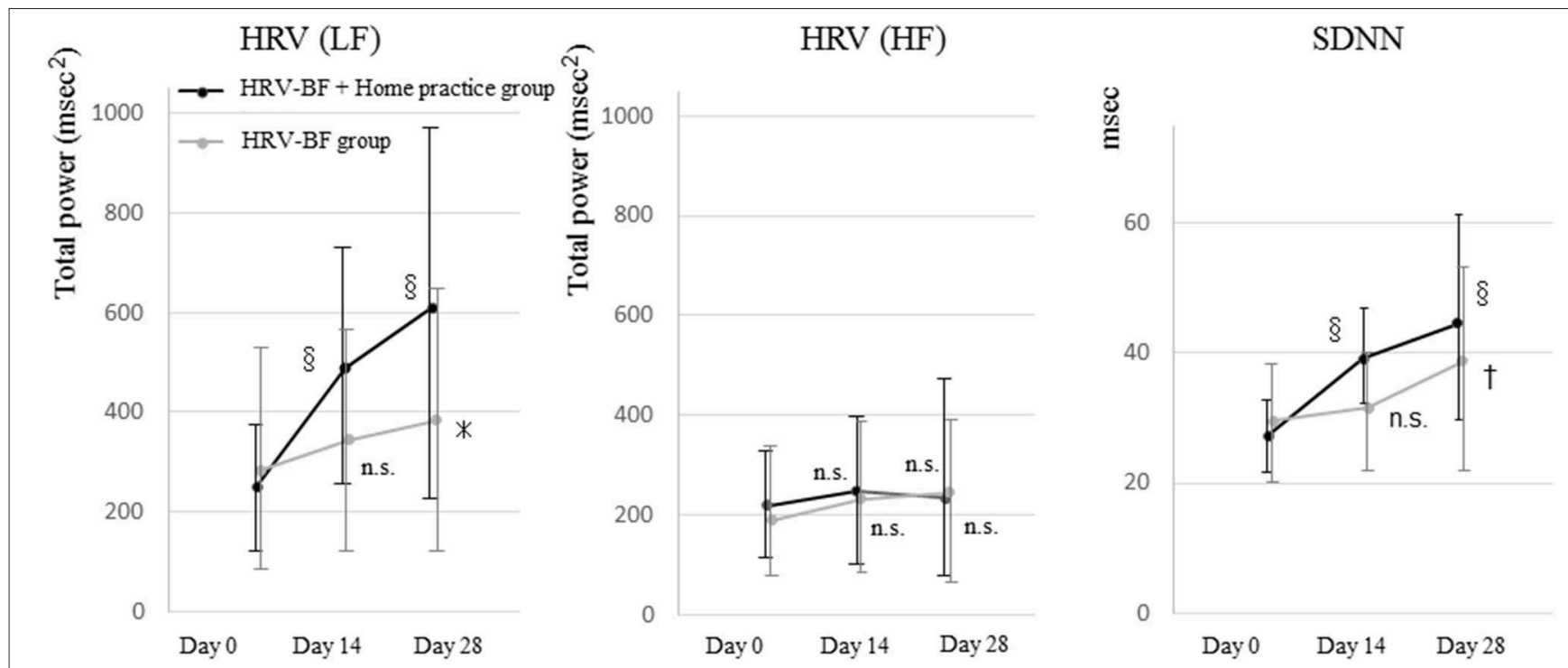

FIGURE 3 | Change in heart rate variability before resonant breathing. HRV, heart rate variability; BF, biofeedback; SDNN, standard deviation of the normal-to-normal interval; LF, low frequency; HF, high frequency; n.s., not significant. ${ }^{*} p<0.05$ (vs. Day 0), ${ }^{\dagger} p<0.01$ (vs. Day 0), ${ }^{8} p<0.001$ (vs. Day 0).

$0, p=0.571$; day $14, p=0.014$; day $28, p=0.001$ ). When we compared SDNN between the groups, there was significant interaction between time course and group $(p=0.043)$, and a significant difference in time course between the groups (day 0 , $p=0.453$; day $14, p=0.010$; day $28, p=0.045$ ). We found no significant difference in HF between the groups, indicating that there was no interaction between time course and group ( $p=$ 0.923) (Figure 3).

The Pearson's correlation coefficient between the variation of PSQI-J total score and the variation of LF score was -0.365 ( $p=$ $0.007)$. The correlation coefficient between the variation of PSQI$\mathrm{J}$ total score and the variation of SDNN score was -0.425 ( $p=$ $0.001)$. There was no correlation between the variation of PSQI-J total score and the variation of HF score $(p=0.484)$.

\section{DISCUSSION}

This study provided the first evaluation report that HRV-BF sessions conducted once every 2 weeks had an effect on sleep among family caregivers with a care burden, and a medium- to short-term effect on autonomic nervous function. In addition, daily practice of RFB at home enhanced this effect. This study targeted family caregivers that had not attended hospitals regularly, but who scored over 30 on the J-ZBI and reported a moderate level of nursing care burden (18). The level of distress among family caregivers of patients with advanced cancer as determined by the prevalence of insomnia disorder was extremely high (52.2\%).

The study results highlighted two important points. First, there may be a positive effect on sleep even with only two sessions of HRV-BF, regardless of whether RFB was practiced at home before bedtime. The total PSQI-J scores in both groups decreased significantly, and showed an improvement in total
PSQI-J score (near 5.5) on Day 28. This study had no control group (without HRV-BF), which was a limitation. However, the LF scores and SDNN scores in the two HRV-BF groups were significantly increased on Day 28, and there was a negative correlation between the variation in PSQI-J total scores and the variation in the LF scores and SDNN scores. It has been reported that LF scores in chronic insomnia patients is lowered during sleep, which is consistent with an increase in sympathetic activity before sleep (26). Therefore, the lowering of the parasympathetic nervous system may be a main pathogenic mechanism for primary insomnia disorder. This suggests that two sessions of HRV-BF had a positive effect on sleep through the interaction between sleep and autonomic nervous function. Previous studies involving HRV-BF sessions compared two time points (before and after the intervention) using 10 or 15 weekly HRV-BF sessions (6-8). Among those studies, one study involving 10 weekly sessions of HRV-BF among patients with depression compared pre-intervention and intervention using 4, 7, and 10 sessions. There was a significant decrease in the Depression Scale Score and an increase in SDNN after four sessions (Day 28) (7), which was similar to our results in terms of a rapidly produced effect. Our results are promising because they suggest that even one HRV-BF session may be feasible for improving skills of family caregivers of patients with cancer, regardless of whether RFB is practiced before bedtime.

The second important point in this study was that daily practice of RFB using a portable device at home enhanced the quality of both sleep and autonomic nervous function over a short period. In particular, the quality of sleep (evaluated by the PSQI-J scores) in the HRV-BF+Home practice group was significantly improved compared with the scores of those in the HRV-BF group on Day 28. Sleep quality was a major primary endpoint in previous studies $(27,28)$. A randomized controlled 
trial involving a HRV-BF group and a control group among 10 healthy persons with experience of polysomnography showed no improvement in sleep latency, but sleep quality was enhanced in the HRV-BF group (11). In addition, the HRV-BF+Home practice group showed a significant increase in LF and SDNN compared with that of the HRV-BF group as early as Day 14 . RFB gives rise to a resonance between breathing and the baroreceptor reflex involving the LF power of HRV (but not HF power of HRV) to increase HRV (5). This suggests that continuous practice of RFB at home indicates a persistent effect, rather than only a transient effect at the time of application. Therefore, because RFB supports an efficient baroreceptor reflex, continuous practice may enhance autonomous homeostasis functions (5). Because there were few reports available for RFB with use of a portable device at home (29), we could not make comparisons with other tests. However, among the PSQI-J component scores, we found that only the numerical values for sleep latency became worse in the HRV-BF+Home practice group. It has been reported that daytime RFB for 2 days could decrease sleep latency, the number of awakenings, and awakening time during sleep, and increase sleep efficiency (29). In contrast, it is assumed that using electronic devices at bedtime could be related to shorter sleeping time, extension of sleep latency, and increased sleep deprivation (30). Therefore, using an unfamiliar electronic device might have influenced sleep latency in our study.

The following items can be described as limitations of the present study. First, we did not objectively evaluate sleep function. There are specific measuring devices available for quantitative sleep parameters at home, such as a threedimensional acceleration device. Using such devices might have further clarified the influence of HRV-BF on sleep. Furthermore, a previous report indicated that HRV-BF before bedtime increased respiratory sinus arrhythmia during sleep (12), but this could not be evaluated in our study because there was no HRV measurement during sleep. Second, this study population consisted of family caregivers of patients with cancer, which made it ethically difficult to include a control group. Therefore, we could not conclude a positive effect on sleep with two sessions of HRV-BF. Since both groups each showed significant improvements, we expected there would be a difference in a group comparison between these groups and a control group. Third, we did not accomplish a long-term evaluation. We found that the HRV-BF sessions produced an effect on the sleep of family caregivers in the medium- to short-term, but did not determine whether such an effect could become persistent. In addition, our follow-up survey indicated that the wearable device

\section{REFERENCES}

1. Maltby KF, Sanderson CR, Lobb EA, Phillips JL. Sleep disturbances in caregivers of patients with advanced cancer: a systematic review. Palliat Support Care. (2017) 15:125-40. doi: 10.1017/S1478951516001024

2. Riemann D, Perlis ML. The treatments of chronic insomnia: a review of benzodiazepine receptor agonists and psychological and behavioral therapies. Sleep Med Rev. (2009) 13:205-14. doi: 10.1016/j.smrv.2008.06.001 at home resulted in insufficient health management in the use group compared with the non-use group, because of a sense of security from using the device (31). Last, because there was a versatility issue because of the single-center design, larger-scale data are required in further studies.

\section{CONCLUSIONS}

Our study suggests that two sessions of HRV-BF could be useful to enhance sleep and autonomic nervous function among family caregivers of patients with cancer. Furthermore, additional actual practice of RFB with use of a portable device at home before bedtime may enhance the effect.

\section{DATA AVAILABILITY STATEMENT}

All datasets generated for this study are included in the article/supplementary material.

\section{ETHICS STATEMENT}

The studies involving human participants were reviewed and approved by the ethics committee of Kansai Medical University, Japan (No. 2015660). The patients/participants provided their written informed consent to participate in this study.

\section{AUTHOR CONTRIBUTIONS}

$\mathrm{HH}$ was responsible for the conception and design of this study and wrote the article, which was critically revised by all the other authors. $\mathrm{HH}$ and MF were responsible for data collection and for clinical evaluations. $\mathrm{HH}$ and $\mathrm{KK}$ were responsible for data analysis. All authors have approved the final version of this manuscript.

\section{FUNDING}

This research was supported by a Yuumi Memorial Foundation for Home Health Care 2016 late Grant.

\section{ACKNOWLEDGMENTS}

The authors thank the palliative care team members of the Kansai Medical University Hospital. We thank Audrey Holmes, MA, from Edanz Group (www.edanzediting.com/ac) for editing a draft of this manuscript. 
5. Lehrer PM. Biofeedback training to increase heart rate variability. In: Lehrer PM, Woolfolk RL, Sime WE, editors. Principles and Practice of Stress Management. 3rd ed. New York, NY: Guilford Press (2007). p 227-48.

6. Karavidas MK, Lehrer PM, Vaschillo E, Vaschillo B, Marin H, Buyske S, et al. Preliminary results of an open label study of heart rate variability biofeedback for the treatment of major depression. Appl Psychophysiol Biofeedback. (2007) 32:19-30. doi: 10.1007/s10484-006-9029-z

7. Chen S, Sun P, Wang S, Lin G, Wang T. Effects of heart rate variability biofeedback on cardiovascular responses and autonomic sympathovagal modulation following stressor tasks in prehypertensives. J Hum Hypertens. (2016) 30:105-11. doi: 10.1038/jhh.2015.27

8. Hassett AL, Radvanski DC, Vaschillo EG, Hassett AL, Radvanski DC, Vaschillo EG, et al. A pilot study of the efficacy of heart rate variability (HRV) biofeedback in patients with fibromyalgia. Appl Psychophysiol Biofeedback. (2007) 32:1-10. doi: 10.1007/s10484-006-9028-0

9. Bernardi L, Porta C, Spicuzza L, Bellwon J, Spadacini G, Frey AW, et al. Slow breathing increases arterial baroreflex sensitivity in patients with chronic heart failure. Circulation. (2002) 105:143-5. doi: 10.1161/hc0202.103311

10. Hayano J, Yasuma F. Hypothesis: respiratory sinus arrhythmia is an intrinsic resting function of cardiopulmonary system. Cardiovasc Res. (2003) 58:1-9. doi: 10.1016/S0008-6363(02)00851-9

11. Bonnet MH, Arand DL. Heart rate variability: sleep stage, time of night, and arousal influences. Electroencephalogr Clin Neurophysiol. (1997) 102:390-6. doi: 10.1016/S0921-884X(96)96070-1

12. Sakakibara M, Hayano J, Oikawa LO, Katsamanis M, Lehrer $P$. Heart rate variability biofeedback improves cardiorespiratory resting function during sleep. Appl Psychophysiol Biofeedback. (2013) 38:265-71. doi: 10.1007/s10484-013-9232-7

13. Ebben MR, Kurbatov V, Pollak CP. Moderating laboratory adaptation with the use of a heart-rate variability biofeedback device (StressEraser). Appl Psychophysiol Biofeedback. (2009) 34:245-9. doi: 10.1007/s10484-009-9086-1

14. McLay RN, Spira JL. Use of a portable biofeedback device to improve insomnia in a combat zone, a case report. Appl Psychophysiol Biofeedback. (2009) 34:319-21. doi: 10.1007/s10484-009-9104-3

15. Brady RE, Constans JI, Marx BP, Spira JL, Gevirtz R, Kimbrell TA, et al. Effect of symptom over-reporting on heart rate variability in veterans with posttraumatic stress disorder. J Trauma Dissociation. (2015) 16:551-62. doi: 10.1080/15299732.2015.1021505

16. Hasuo H, Kanbara K, Sakuma H, Yoshida K, Uchitani K, Fukunaga M. Selfcare system for family caregivers of cancer patients using resonant breathing with a portable home device: a randomized open-label study. J Palliat Med. (2018) 22:18-24. doi: 10.1089/jpm.2018.0230

17. Hall M, Vasko R, Buysse D, Ombao H, Chen Q, Cashmere JD, et al. Acute stress affects heart rate variability during sleep. Psychosom Med. (2004) 66:5662. doi: 10.1097/01.PSY.0000106884.58744.09

18. Schreiner AS, Morimoto T, Arai Y, Zarit S. Assessing family caregiver's mental health using a statistically derived cut-off score for the Zarit Burden Interview. Aging Ment Health. (2006) 10:107-11. doi: 10.1080/13607860500312142

19. Tarvainen MP, Niskanen JP, Lipponen JA, Ranta-Aho PO, Karjalainen PA. Kubios HRV-heart rate variability analysis software. Comput Methods Programs Biomed. (2014) 113:210-20. doi: 10.1016/j.cmpb.2013.07.024
20. Sookan T, McKune AJ. Heart rate variability in physically active individuals: reliability and gender characteristics. Cardiovasc J Afr. (2012) 23:67-72. doi: 10.5830/CVJA-2011.108

21. American Psychiatric Association. Diagnostic and Statistical Manual of Mental Disorders. 5th ed. Arlington, VA: American Psychiatric Publishing (2013).

22. Carney S, Koetters T, Cho M, West C, Paul SM, Dunn L, et al. Differences in sleep disturbance parameters between oncology outpatients and their family caregivers. J Clin Oncol. (2011) 29:1001-6. doi: 10.1200/JCO.2010.30.9104

23. Montano N, Ruscone TG, Porta A, Lombardi F, Pagani M, Malliani, A. Power spectrum analysis of heart rate variability to assess the changes in sympathovagal balance during graded orthostatic tilt. Circulation. (1994) 90:1826-31. doi: 10.1161/01.CIR.90.4.1826

24. Task Force of the European Society of Cardiology and the North American Society of Pacing and Electrophysiology. Heart rate Variability. Standards of measurement, physiological interpretation, and clinical use. Eur Heart J. (1996) 17:354-81.

25. Nunan D, Sandercock GR, Brodie DA. A quantitative systematic review of normal values for short-term heart rate variability in healthy adults. Pacing Clin Electrophysiol. (2010) 33:1407-17. doi: 10.1111/j.1540-8159.2010.0 2841.x

26. Farina B, Dittoni S, Colicchio S, Testani E, Losurdo A, Gnoni V, et al. Heart rate and heart rate variability modification in chronic insomnia patients. Behav Sleep Med. (2014) 12:290-306. doi: 10.1080/15402002.2013.801346

27. Hoey LM, Fulbrook P, Douglas JA. Sleep assessment of hospitalised patients: a literature review. Int J Nurs Stud. (2014) 51:1281-8. doi: 10.1016/j.ijnurstu.2014.02.001

28. Yeung V, Sharpe L, Glozier N, Hackett ML, Colagiuri B. A systematic review and meta-analysis of placebo versus no treatment for insomnia symptoms. Sleep Med Rev. (2018) 38:17-27. doi: 10.1016/j.smrv.2017.03.006

29. Tsai HJ, Kuo TB, Lee GS, Yang CC. Efficacy of paced breathing for insomnia: enhances vagal activity and improves sleep quality. Psychophysiology. (2015) 52:388-96. doi: 10.1111/psyp.12333

30. Hysing M, Pallesen S, Stormark KM, Jakobsen R, Lundervold AJ, Sivertsen B. Sleep and use of electronic devices in adolescence: results from a large population-based study. BMJ Open. (2015) 5:e006748. doi: 10.1136/bmjopen-2014-006748

31. Jakicic JM, Davis KK, Rogers RJ, King WC, Marcus MD, Helsel D, et al. Effect of wearable technology combined with a lifestyle intervention on long-term weight loss: the IDEA randomized clinical trial. JAMA. (2000) 316:1161-71. doi: 10.1001/jama.2016.12858

Conflict of Interest: The authors declare that the research was conducted in the absence of any commercial or financial relationships that could be construed as a potential conflict of interest.

Copyright $\odot 2020$ Hasuo, Kanbara and Fukunaga. This is an open-access article distributed under the terms of the Creative Commons Attribution License (CC BY). The use, distribution or reproduction in other forums is permitted, provided the original author(s) and the copyright owner(s) are credited and that the original publication in this journal is cited, in accordance with accepted academic practice. No use, distribution or reproduction is permitted which does not comply with these terms. 